\title{
Transferability of giant atomic clusters in the HCP crystals twinning
}

Shan Jiang ${ }^{a}$

Research Institute for New Materials and Technology, Chongqing University of Arts and Sciences, No. 329, Hong he Road, Yongchuan District 402160, China

a382595277@qq.com

Keywords: HCP crystals; Deformation; Twinning; Atomic motion; Mechanism.

\begin{abstract}
The atomic motions in the twinning deformation of hexagonal close-packed (HCP) crystals was investigated at the atomic scale by using the giant atomic clusters (GACs) induced model, aimed at deepening the understanding to the twinning mechanism. It is found that the twin boundaries are composed of gradually oriented GACs with their orientation between the matrix and the twin, and the GACs can transfer their rotation under adequate stress, which supplies a reasonable explanation for the occurrence of twinning. These findings are relevant for understanding the underlying origin behind many intriguing phenomena in the HCP crystals.
\end{abstract}

\section{Introduction}

As the light structural metal materials with superior specific stiffness and strength, HCP crystal metals (e.g. magnesium and titanium alloys) are of intensive researches because of the hopes it raises for a variety of engineering applications and also for its fundamental scientific importance [1-3] Though many studies have been conducted on deformation of HCP crystal materials [4-7], origin of their poor mechanical properties has not yet been well understood and remains a key puzzle for these engineering materials. In view of its ubiquity, mechanical twinning is intuitively viewed as an important deformation mode for the materials and thus has attracted a broad attention. Extensive experiments verify that it is twinning that dominates deformation at low temperatures [8-9], taking into account the fact that twinning can shift abruptly orientation of grains from that of their basals, which may reactivate other slip systems.

Though a number of intriguing phenomena have been observed concerning twins in $\mathrm{Mg}$ alloys, for example, the twin growth, mutual interception between twins, detwinning, and recrystallization in twin interiors [10-11], the deformation mechanism triggered by twins in $\mathrm{Mg}$ alloys at room temperature has not been well clarified yet because of the complications in a direct observation of twin evolvement in experiment, together with the lack of theoretical models. In addition, it is still unknown what kind of atomic motion (e.g., microstructure change) emerges as twins behaves and how the twinning affects the tensile plastic deformation and texture formation. These points remain unclear because of the following reason: twinning occurs at intermediate states, but current studies on the deformation of HCP materials are generally restricted to initial and final states only.

Unexpectedly, we have found that the atomic motion in HCP crystals twinning can be reasonably addressed by the GAC model, i.e. the transference of the GACs under sustained strain realizes the rotation of GACs and the occurrence of twinning. 


\section{Modelling procedures}

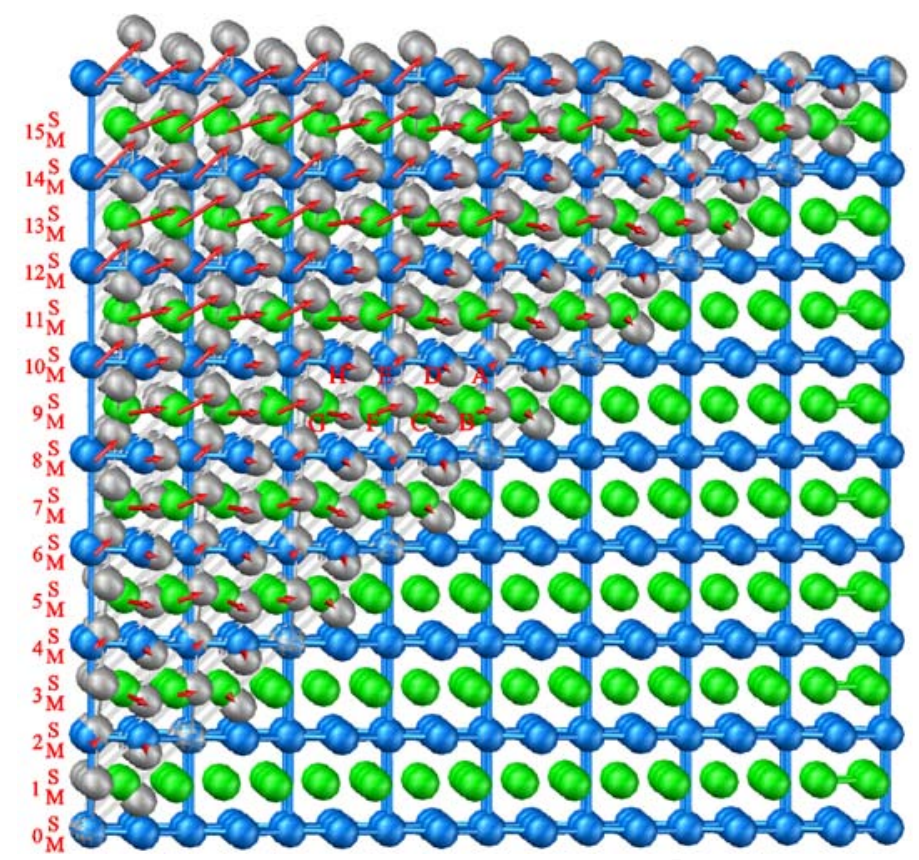

Fig. 1 Schematic of the atomic motion in the $\{1012\}$ twinning of HCP crystals.

Figure 1 presents the atomic motion of $\{10 \overline{1} 2\}$ twinning in the HCP crystals. For a better description, the crystal planes are classified into two species according to the stacking sequence $\mathrm{ABAB} \ldots$ in the HCP structure: (i) main planes (labeled M), and (ii) sub-planes (S), which are defined as the planes passing through $\mathrm{A}$ and B-layer atoms, respectively. And the $\{10 \overline{1} 2\}$ crystal planes are numbered according to their distance to the twin boundary: the pair of plane located at the $\{10 \overline{1} 2\}$ twin boundary are numbered as " 0 " and from which to the distant " $0,1,2, \ldots k$ ". Thus the $k$-th main plane can be expressed as the plane $M_{k}$ (others similar). Because the "shearing" atoms appear repeatedly in every four planes and the atoms in one twinning plane move in the same way, only a part of representative atoms are selected to discuss.

The parallelogram " $A B C D$ " composed of given atoms $A, B, C$ and $D$ is selected to be a specific research area. Note that atom $A$ is a shearing atom while the others are shuffling atoms. The final position of the twinning atoms are determined by the symmetric relation between the twin and the matrix. To simplify the space vector calculation of the twinning atoms, a three-dimensional Cartesian coordinate system is built in a HCP crystal cell as shown in the lower right of Fig. 1. Thus the displacement vectors of the twinning atoms are expressed as follows:

$$
\begin{aligned}
& \vec{v}_{A}=\vec{v}_{M 2}=\Delta x_{A} i+\Delta y_{A} j+\Delta z_{A} k \\
& \vec{v}_{B}=\vec{v}_{S 1}=\Delta x_{B} i+\Delta y_{B} j+\Delta z_{B} k \\
& \vec{v}_{C}=\vec{v}_{S 2}=\Delta x_{C} i+\Delta y_{C} j+\Delta z_{I} k \\
& \vec{v}_{D}=\vec{v}_{M 3}=\Delta x_{D} i+\Delta y_{D} j+\Delta z_{D} k
\end{aligned}
$$

where $\vec{v}_{A}$ is the displacement vector of atom $M ; \vec{v}_{M 2}$ represents the displacement vector of atoms in the plane $M_{2}$; and $\Delta x_{A}$ is the vector components of atom $A$ along $x$ axis (others similar).

Then the shearing atoms satisfy the following relation:

$$
\vec{v}_{M(2 k+2)}=(k+1) \vec{v}_{A}=(k+1) \vec{v}_{M 2} \quad(k=0,1, \ldots)
$$

Here $\vec{v}_{A}$ is the minimum shearing vector of all and serves as a unit vector.

Similarly, the shuffling atoms satisfy the following relation:

$$
\vec{v}_{M(2 k+1)}=\vec{v}_{M I}+k \vec{v}_{A} \quad(k=0,1, \ldots)
$$




$$
\begin{array}{ll}
\vec{v}_{S(2 k+1)}=\vec{v}_{S 1}+k \vec{v}_{A} & (k=0,1, \ldots) \\
\vec{v}_{S(2 k+2)}=\vec{v}_{S 2}+k \vec{v}_{A} & (k=0,1, \ldots)
\end{array}
$$

Then above Eq. (5), (6), (7) and (8) can then be further combined as the follows:

$$
\begin{aligned}
& \vec{v}_{M(2 k+i)}=\vec{v}_{M i}+k \vec{v}_{A} \\
& \vec{v}_{S(2 k+i)}=\vec{v}_{S i}+k \vec{v}_{A}
\end{aligned}
$$

where $i=1,2 ; k=0,1,2 \ldots n$ ( $n$ is the sum of the twinning planes in the twin). Eq. (9) and (10) indicate that the twining atoms present a good periodicity.

The location of the twinning atoms of some GACs before and after the $\{10 \overline{1} 2\}$ twinning is illustrated in Fig. 2, where the displacement vectors for mobile atoms are labeled. When considered as a whole, the parallelogram $A B C D$ is found to make a combined motion of shifting for a distance of $\left|\vec{v}_{A}\right|$ along the shearing direction and rotation for an angle of $\alpha$ round the atoms $A$ in counter-clockwise direction. Evidently, this case applies to the whole twinning area, i.e., each twinning atom can be grouped into a parallelogram grouped by four specified atoms (seen from the two-dimensional image) that belong to the planes $M_{(2 \mathrm{k}+2)}, M_{(2 \mathrm{k}+1)}, S_{(2 \mathrm{k}+1)}$ and $S_{(2 \mathrm{k}+2)}$, which makes a shifting of $\left|(k+1) \vec{v}_{A}\right|$ and a rotation angle of $\alpha$.

Because each atom of the above parallelograms represents a line of close-packed atoms along the $x$-axis, the actual space structure of parallelogram $A B C D$ should be composed of massive octahedrons with their edges connected. For this reason, this special structure is named "giant atomic clusters (GACs)" here. Based on the above analysis, we can make a reasonable speculation that the twinning is induced by the rotation of the GACs.

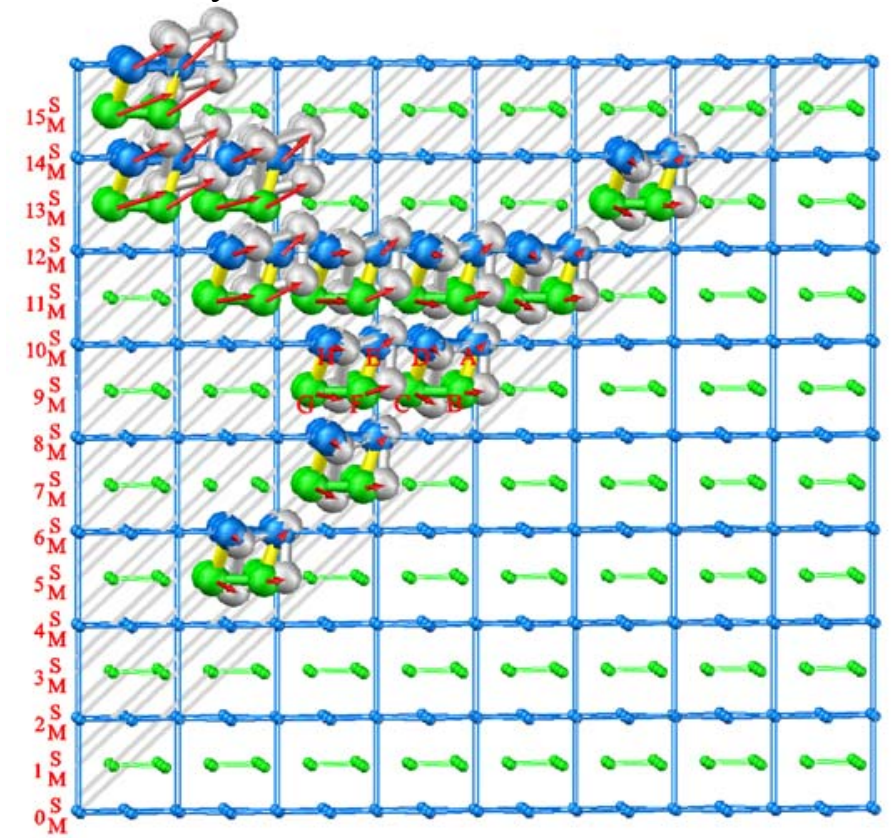

Fig. 2 Illustration of the GACs induced mechanism in the $\{10 \overline{1} 2\}$ twinning of HCP crystals.

\section{Discussion}

It was considered earlier that some atoms undertake shear motion while the others reconstruct during twinning and that intensity of shear in twinning and ratio of shearing atoms to all twinning atoms play a key role in promoting the twinning [12]. However, it is still difficult to figure out how reconstructed atoms displace based on this theory. In addition, how the atoms belonging to different subareas are connected and interacted during twinning remains unclear.

In a separate paper, we have developed the GACs induced model to uncover atomic motions in the HCP crystals twinning [13-15]. Following this model, these twinning modes can be realized by rotating the GACs as a whole. Here, we systematically clarify the transfer mechanism between 
GACs and expand the GACs to twin boundaries, growth, and detwinning. Figure 3 shows the arrangement of the GACs during the $\{10 \overline{1} 2\}$ twinning, where it can be seen that the GACs are arranged periodically. In addition, the rotations of GACs are continuous and transferable.

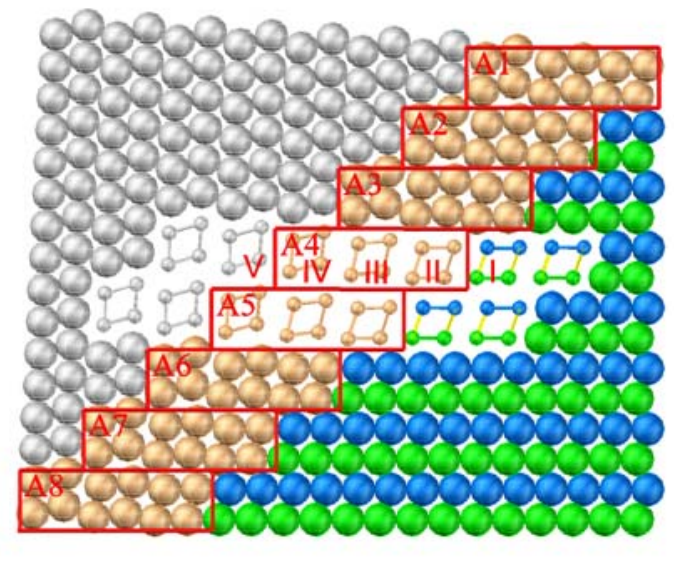

Fig. 3 Schematic illustration of the distorted GACs arrangements distributed at the $\{10 \overline{1} 2\}$ twin boundary.

As aforementioned, it is frequently observed both twinning and detwinning in $\mathrm{Mg}$ alloys, which can be attributed to the motion of twin boundaries outward and inward, respectively. Note that the motion of twin boundaries differs from GB sliding in that it is perpendicular to the interface. Since twining can be understood as a process of the rotation of GACs, we speculate that the twin boundaries are composed of the GACs oriented gradually between the matrix and the twin, and the GACs around twin boundary are repeated periodically both along ( $A_{1}$ to $\left.A_{8}\right)$ and across ( $I$ to $V$ ) the twinning direction (Fig. 3), which together favors the transferability of GACs for rotation. When a twin boundary is in stress equilibrium, the GACs keep still. As soon as the stress equilibrium is destroyed, the GACs at the twin boundary begin to rotate, resulting in the migration of the twin boundary. As a specific case (Fig 3), when the GACs rotate counterclockwise, the twin boundary migrates to the right side and the twin grows.

\section{Conclusions}

The atomic motions in the twinning deformation of HCP crystals is investigated by using the GAC model at the atomic scale. It is found that the twin boundaries are composed of gradually oriented GACs with their orientation between the matrix and the twin, and the GACs can transfer their rotation under adequate stress, which supplies a reasonable explanation for the occurrence of twinning. These findings are relevant for understanding of underlying origin behind many intriguing phenomena in the HCP crystals.

\section{Acknowledgements}

This work is supported by National Natural Science Foundation of China (No. 51301215), Natural Science Foundation Project of Chongqing (No. cstc2012jjA 50016), Talent project of Chongqing University of Arts and Sciences (R2014CJ04; Y2014CJ29), and Chongqing Post-doctor Support Program (Rc201334).

\section{References}

[1] Y. N. Wang, J. C. Huang. The role of twinning and untwinning in yielding behavior in hot-extruded Mg-Al-Zn alloy. Acta Mater. 55 (2007) 3, 897-905.

[2] A. Yamashita, Z. Horita, T. G. Langdon. Improving the mechanical properties of magnesium and a magnesium alloy through severe plastic deformation. Mater. Sci. Eng. A. 300 (2001)1-2, 142-147. 
[3] C. N. Tomé, R. A. Lebensohn, U. F. Kocks. A model for texture development dominated by deformation twinning: Application to zirconium alloys. Acta Metall. Mater. 39 (1991) 11, 2667-2680.

[4] A. Serra, D. J. Bacon. A new model for $\{10-12\}$ twin growth in hep metals. Phil. Mag. A. 73 (1996) 333-343.

[5] A. Serra, D. J. Bacon. Computer simulation of screw dislocation interactions with twin boundaries in H.C.P. metals. Acta Metall. Mater. 43 (1995) 12, 4465-4481.

[6] T. Liu, Y. D. Wang, S. D. Wu, et al. Textures and mechanical behavior of Mg-3.3\%Li alloy after ECAP. Scripta Mater. 51 (2004) 11, 1057-1061.

[7] K. Xia, J. T. Wang, X. Wu, G. et al. Equal channel angular pressing of magnesium alloy AZ31. Mater. Sci. Eng. A. 410 (2005) 25, 324-327.

[8] X. Y. Lou, M. Li, R. K. Boger, S. R. Agnew, R. H. Wagoner: Int. J. Plasticity 23 (2007) 44-86.

[9] S. G. Hong, H. P. Sung, C. S. Lee. Role of $\{10-12\}$ twinning characteristics in the deformation behavior of a polycrystalline magnesium alloy. Acta Mater. 58 (2010) 18, 5873-5885.

[10] S. M. Yin, F. Yang, X. M. Yang, et al. The role of twinning-detwinning on fatigue fracture morphology of Mg-3\%Al-1\%Zn alloy. Mater. Sci. Eng. A 494 (2008)1-2, 397-400.

[11] T. Al-Samman, G. Gottstein. Dynamic recrystallization during high temperature deformation of magnesium. Mater. Sci. Eng. A. 490 (2008) 1-2, 411-420.

[12] M. A. Jaswon, D. B. Dove. The crystallography of deformation twinning. Acta Cryst. 13 (1960) $232-240$.

[13] S. Jiang, T. Liu, L. Lu, et al. Atomic motion in Mg-3Al-1Zn during twinning deformation. Scripta Mater. 62 (2010) 556-559.

[14] S. Jiang, T. Liu, C. Chen. Law of Atomic Motion during f10 $7111 \mathrm{~g}$ Twinning in Magnesium Alloys. Mater. Trans. 52 (2011) 1585-1588.

[15] S. Jiang. Atomic Group Rotation Mechanism for $\mathrm{f} 10\urcorner 12 \mathrm{~g}$ Twinning of HCP Crystal Materials. Mater. Trans. 55 (2014) 907 -910. 